\title{
Vorschläge für eine bedarfsorientierte Neuordnung des Finanzausgleichs
}

\author{
Nathalie Behnke
}

\section{Einleitung}

Der deutsche Föderalismus wird häufig als unitarischer Bundesstaat beschrieben (Hesse 1962; Lehmbruch 2002). Die Länder besitzen zwar einerseits starke Kompetenzen der Selbst- und der Mitentscheidung; in der Praxis kommen aber dem Bund weitreichende Regelungskompetenzen zu, weil über eine wichtige normative Prämisse ein breit geteilter Konsens zwischen Bund und Ländern besteht: Im gesamten Bundesgebiet sollen gleichwertige Lebensverhältnisse herrschen, und die Rechts- und Wirtschaftseinheit soll gewahrt bleiben. Soweit eine Regulierung durch den Bund nachweislich diesem Ziel dient, sind die Länder bereit, im Rahmen der konkurrierenden Gesetzgebung diese Kompetenz dem Bund auch zuzugestehen (Art. 72 Abs. 2 GG). Diese Grundkonzeption erstreckt sich nicht nur auf die Gesetzgebungskompetenzen, sondern auch auf die Finanzverfassung. Zwar haben die Länder gewisse autonome steuerliche Einnahmequellen, um unabhängig wirtschaften zu können (allerdings haben sie praktisch keine Kompetenzen in der Steuergesetzgebung, s. Art. 104 Abs. 2a GG); die aufkommensstarken Steuern werden aber im Verbund vereinnahmt und dann zwischen den Gebietskörperschaften verteilt. Auch hier gilt wieder explizit das Ziel, dass die Einheitlichkeit der Lebensverhältnisse im Bundesgebiet gewahrt bleibt (Art. 106 Abs. 3, Satz 4).

Man orientiert sich am Konzept der Finanzkraft als entscheidendem Kriterium zur Feststellung der Leistungsfähigkeit der Länder (Art. 107 Abs. 2 sowie $\S 6$ FAG). Denn wenn alle Länder über eine vergleichbare Finanzkraft verfügen, so die Annahme, dann sind sie auch in vergleichbarem Maße in der Lage, ihre Aufgaben zu erfüllen und ihre Ausgaben zu decken. Die Finanzkraft wird ermittelt auf Basis der Steuereinnahmen eines Landes gemäß § 7 FAG. Im bestehenden Finanzausgleichssystem wird dann über mehrere Ausgleichsschritte eine weitgehende Angleichung dieser Finanzkraft erreicht. Auf diese Weise sollen die aufgabenangemessene 
Finanzausstattung der Länder und damit die Herstellung gleichwertiger Lebensverhältnisse im Bundesgebiet gesichert werden. ${ }^{1}$

Das bestehende Finanzausgleichssystem wählt also eine spezifische Kombination von Elementen, die einerseits die Autonomie der Länder sichern und die Finanzausstattung der Länder an ihre eigene finanzielle Leistungsfähigkeit koppeln und andererseits durch solidarische Umverteilung die vergleichbare Aufgabenerfüllung durch die Länder im gesamten Bundesgebiet sicherstellen soll, auch wenn deren eigene finanzielle Leistungsfähigkeit unterdurchschnittlich ist. Die meisten Kritiken und auch Reformvorschläge orientieren sich zunächst an dieser bestehenden Kombination von Elementen, ohne die grundlegende Architektur des Systems in Frage zu stellen, versuchen also gewissermaßen die Symptome zu kurieren. Ein Verständnis für die Logik dieser einzelnen Bausteine eröffnet aber den Weg für eine wesentlich grundsätzlichere Debatte, in der man sich zum einen über das Verhältnis zwischen Autonomie und Solidarität (oder Leistungskomponente vs. Umverteilung) politisch verständigt und zum anderen über die geeigneten Formen nachdenkt, in denen beide Prinzipien in angemessenen Anteilen instrumentell in den föderalen Finanzbeziehungen verwirklicht werden können.

Der hier präsentierte Reformvorschlag reiht sich in eine Gruppe von Gutachten und Vorschlägen ein, die in den letzten Jahren formuliert wurden und die alle mehr oder weniger weitgehend diese Grundarchitektur hinterfragen (Heinemann 2013; Eichel et al. 2013; Knöchel, et al. 2014; Behnke 2012; Wieland 2012). Die Hauptstoßrichtung dieser Reformvorschläge zielt darauf, das bestehende Finanzausgleichssystem auf die Finanzkraft auszurichten, und schlägt vor, die Finanzkraftorientierung durch eine stärkere Bedarfsberücksichtigung in der Steuerverteilung zu ergänzen oder gar zu ersetzen. Die Notwendigkeit, eine stärkere Bedarfsorientierung im Finanzausgleichssystem zu verankern, dürfte mittlerweile kaum mehr bestritten werden. ${ }^{2}$ Die Frage ist dann aber, welche Konsequenzen

1 Wenn im Folgenden von dem ,aktuellen“' oder ,geltenden“" Finanzausgleichssystem die Rede ist, ist damit immer das Finanzausgleichsgesetz (FAG) in der Fassung der Bekanntmachung durch Art. 5 des Gesetzes vom 20.12.2001 (BGBl. I S. 3955) mit den jeweils aktuellen Änderungen gemeint. Dieses Gesetz gilt seit dem 01. Januar 2005 und tritt zum 31.12.2019 außer Kraft.

2 Tatsächlich enthält auch das bestehende FAG eine Reihe von Abweichungen von der Finanzkraftorientierung. Hier werden die Bedarfe allerdings relativ unsystematisch berücksichtigt. Dies verwundert nicht, stellen sich diese Ergänzungen 
man aus diesem Befund zieht. Man kann einerseits versuchen, innerhalb des bestehenden Zuteilungssystems den Anteil der Bedarfskomponenten zu erhöhen und diese passgenauer auf regional stark unterschiedliche Ausgabenbelastungen zuzuschneiden. Oder man kann auf die Mühe verzichten, eine möglichst ausgabenadäquate Finanzausstattung der Länder im Rahmen komplizierter Umverteilungsverfahren zu erreichen, und stattdessen dem Bund direkt die finanziellen Mittel und die Kompetenzen zuweisen, diese Aufgaben sozusagen in direkter Rechnung zu begleichen. Letzterer Vorschlag zielt auf eine so genannte Vertikalisierung des Finanzausgleichs bzw. der Ausgabenverpflichtung. Zwischen diesen beiden Polen - einer möglichst aufgabenadäquaten Ausstattung der Länder einerseits und einer weitgehenden Vertikalisierung andererseits - muss ein Finanzausgleichssystem sich positionieren. Die Entscheidung, welches Mischungsverhältnis von zentraler versus dezentraler Finanzausstattung einerseits und von finanzkraft- versus bedarfsorientierter Zuweisung der Finanzmittel andererseits man wählt, muss politisch getroffen werden. Hierfür kann keine Simulation von Verteilungseffekten einen eindeutigen Hinweis liefern.

Mein Reformvorschlag, den ich in diesem Beitrag präsentiere, zielt ebenfalls auf ein anderes Mischungsverhältnis zwischen Finanzkraft- und Bedarfsorientierung und verortet sich zwischen den genannten Polen. Der im Folgenden vorgeschlagene Modus der horizontalen Steuerverteilung dient einer Stärkung der Bedarfskomponente und mag bei oberflächlichem Lesen den Eindruck erwecken, ich würde eine stärkere Vertikalisierung des Finanzausgleichs befürworten (vgl. Korioth 2009). Tatsächlich zielt aber die vorgeschlagene Veränderung in der vertikalen Steuerverteilung auf eine Stärkung der Fiskalautonomie der Länder, wohingegen in der horizontalen Verteilung das Leistungsprinzip in seiner Bedeutung reduziert wird. Um diesen Vorschlag einzuführen und zu begründen, werden zunächst drei grundlegende Diskussionen über diejenigen Prinzipien geführt, die dem bestehenden System zugrunde liegen. In Abschnitt 2 wird das Gegensatzpaar Autonomie versus Solidarität vorgestellt, und es wird ver-

doch in der historischen Betrachtung als das Ergebnis von Koppelgeschäften in wiederholten Reformrunden dar. Darüber hinaus ist aber auch der Ansatz abzulehnen, solche Bedarfe mit Hilfe diskreter Zuteilungsformeln anzurechnen. Demgegenüber ist eine kontinuierliche Funktion klar vorzuziehen (vgl. die entsprechende Diskussion in Behnke 2012, S. 54). 
deutlicht, dass diese Prinzipien nicht nur im horizontalen Verhältnis der Länder untereinander relevant sind, sondern auch für die vertikale Beziehung zwischen der Länder- und der Bundesebene eine wichtige Rolle spielen. In Abschnitt 3 wird hinterfragt, wodurch denn das „Eigene“ in der Steuerverteilung definiert wird. Es wird gezeigt, dass die verfügbare Steuermasse einer Gebietskörperschaft maßgeblich durch Steuergesetze definiert wird, wobei insbesondere die Steuerzerlegung eine prominente Rolle spielt. Diese Definitionen sind nicht zwangsläufig, und insofern kann sich aus einem definierten „örtlichen Aufkommen“ auch schwerlich ein normativer Verfügungsanspruch über diese Mittel begründen. Schließlich wird in Abschnitt 4 das Konzept der Finanzkraft als Maßstab der Finanz(um-) verteilung kritisch erörtert. Die zugrundeliegende Annahme besteht darin, dass eine gleiche Finanzkraft auch eine gleiche Aufgabenerfüllung gewährleistet. Eine solche Annahme negiert aber die Möglichkeit, dass Bürger aufgrund struktureller Bedingungen für Leistungen regional unterschiedliche Kosten verursachen. Diesen Effekt kann man nur durch strukturelle Bedarfsindikatoren angemessen kompensieren. In Abschnitt 5 wird dann ein Reformvorschlag skizziert, der die hier ausgearbeiteten Kritikpunkte aufgreift und umsetzt. Es geht hierbei noch nicht um konkrete Zahlen, sondern um die Skizze einer neuen Architektur der föderalen Finanzbeziehungen, die einige grundlegende Defizite des bestehenden Systems überwinden könnte. Jeder Reformvorschlag bleibt angesichts der Komplexität der realen finanziellen Verflechtung zwischen den Ebenen notwendig Stückwerk. Daher werden in Abschnitt 6 einige weitere Problemkreise angedeutet, die in einer umfassenden Reformdebatte berücksichtigt werden müssen.

\section{Zum Verhältnis zwischen Autonomie und Solidarprinzip}

Das Finanzausgleichssystem in Deutschland stellt eine sorgfältig ausbalancierte Kombination aus Elementen des Autonomieprinzips und des Solidarprinzips dar. In seiner grundgesetzlichen Ausformung bezieht sich das Prinzip der Autonomie primär auf die vertikale Dimension, konstatiert also eine Autonomie der Ebene der Länder gegenüber der Ebene des Bundes. Es findet sich in der Feststellung der Eigenstaatlichkeit der Länder (Art. 30 GG), in der Haushaltsautonomie der Länder (Art. 109 Abs. 1 GG) sowie im Konnexitätsprinzip (Art. 104a Abs. 1 GG). Praktisch bedeutet die Kombination dieser Prinzipien, dass die Länder für diejenigen Aufga- 
ben, die sie erfüllen, finanziell auch selbst aufkommen müssen. Daraus abgeleitet folgt, dass sie hierfür ausreichend finanzielle Mittel zur Verfügung haben müssen. Hier bekommt das Autonomieprinzip eine horizontale Dimension, indem nach der Logik des "örtlichen Aufkommens“ (Art. 107 Abs. 1 GG) Steuereinnahmen erst einmal demjenigen Land zustehen, in dem sie vereinnahmt werden. Die Generierung von Steuereinnahmen wird als eine eigene politische Leistung interpretiert, deren Früchte auch denjenigen zu Gute kommen sollen, die die Leistung erbracht haben.

Auch das Solidarprinzip hat eine vertikale und eine horizontale Dimension. In seiner vertikalen Dimension ist es grundgesetzlich im Prinzip „Einheitlichkeit der Lebensverhältnisse“ (Art. 106 Abs. 3 Punkt 2 GG) als Grundsatz für Umsatzsteuerverteilung zwischen Bund und Ländern nach dem so genannten „Deckungsquotenprinzip“ konkretisiert. Auch die Bundesergänzungszuweisungen realisieren das vertikale Solidarprinzip. In der horizontalen Dimension findet das Solidarprinzip in der Verteilung der Umsatzsteueranteile zwischen den Ländern sowie im Länderfinanzausgleich (Art. 107 Abs. 2 GG) Anwendung. Die Anwendung des Solidarprinzips stellt sicher, dass die finanzielle Ausstattung der Länder so weit angenähert wird, dass das Angebot an öffentlichen Leistungen im Bundesgebiet überall vergleichbaren Standards genügt.

Das Autonomieprinzip in der vertikalen Dimension ist eine logische Folge des Föderalismusprinzips. Für eine dezentrale Finanzausstattung und Fiskalkompetenz spricht zuallererst das Föderalismusprinzip per se: "If one level of government has the money and another is always begging for it, cap in hand, no constitutional principle of independence or autonomy is going to change the practical fact of dependency" (Bakvis et al. 2009, S. 137). Es ist ein Grundbedürfnis autonomer Gebietskörperschaften, auch über eigene Finanzmittel zu verfügen, die einem nicht nur von einer zentralen Ebene zugewiesen werden. Diese Diskussion sieht man besonders gut in UK, wo seit vier Jahren eine intensive Debatte um die Reform des finanziellen Zuweisungssystems (,Block grants“ nach der so genannten „Barnett formula“) im Gange ist, das den erweiterten autonomen Kompetenzen in den Devolutionsregionen Schottland und Wales einfach nicht mehr angemessen ist (Trench 2013). Für eine dezentrale Ressourcenverantwortung spricht darüber hinaus das Ziel der wirtschaftlichen Haushaltsführung. Würden Kosten, die bei Ländern oder Kommunen im Vollzug anfallen, komplett vom Bund erstattet, wie dies etwa für kommunale Sozialausgaben die Reformmodelle der LINKEN und der FES empfehlen (vgl. Knöchel et al. 2014; Eichel et al. 2013), fiele jeder Anreiz zum 
sparsamen Wirtschaften weg. Auch entfiele dann eine demokratische Rückkopplung der Verantwortlichkeit gegenüber den Wählern.

Auch in der horizontalen Dimension der Autonomie ist es wichtig, dass sowohl auf der Einnahme- als auch auf der Ausgabeseite eine Eigenverantwortlichkeit für die verfügbaren Ressourcen und eine erkennbare Koppelung von Eigenleistung an Erträge bestehen. Diese Koppelung von Eigenleistung an Erträge ist allerdings nicht im neoliberalen Sinne so zu verstehen, dass alles, was auf einem Territorium erwirtschaftet wird, nur und unmittelbar durch die Leistung der Erwirtschaftenden zustande gekommen sei (vgl. die Diskussion zum „Eigenen“ im folgenden Abschnitt). In diesem Sinne überzeugt auch das häufig vorgebrachte Argument, man müsse den Wettbewerb zwischen Ländern und Kommunen stärken (vgl. Feld et al. 2013) nicht. Denn worum genau soll konkurriert werden? Der Dumping-Wettbewerb der Steuersätze um Steuerzahler erbringt vorhersehbar nach der Logik des Gefangenendilemmas für alle Gebietskörperschaften langfristig nur Mindereinnahmen. Ein Wettbewerb um bessere Wirtschafts- und Standortpolitik hingegen überschätzt bei Weitem die Möglichkeiten der Politik zur aktiven Beeinflussung und Steuerung wirtschaftliche Prozesse. Diese sind in erster Linie von gesamtkonjunkturellen Entwicklungen abhängig, die sich ohnehin dem Einfluss lokaler Politik entziehen. Darüber hinaus sind sie, wie im folgenden Abschnitt ausgeführt wird, einerseits von rechtlichen Zuschreibungen und andererseits von Entscheidungen der Vergangenheit abhängig. Die Ausgabesituation schließlich ist, wie im übernächsten Abschnitt diskutiert wird, zumindest zu einem großen Teil von strukturellen Bedingungen abhängig, auf die die Regierung des Tages ebenfalls wenig Einfluss hat. Insofern geht ein Argument, das behauptet, Länder könnten um Steuereinnahmen, um Wirtschaftsunternehmen oder auch um bessere Politiken konkurrieren, zu einem großen Teil von falschen Annahmen aus. Vielmehr muss in einem sehr grundlegenden Sinne eine Koppelung von Eigenleistung an Erträge darin bestehen, dass beispielsweise Kommunen davon profitieren, wenn es ihnen gelingt, Wirtschaftsunternehmen anzusiedeln; für Länder sollte es keinen finanziellen Nachteil bringen, wenn sie genügend Steuerprüfer einstellen; und der sparsame Vollzug sollte dadurch angereizt werden, dass das Ersparte dann auch für andere Ausgaben zur Verfügung steht. In diesem Sinne trägt eine vertikale und horizontale Finanzautonomie dazu bei, dass eine grundlegende Leistungsmotivation gesichert und das Selbstwertgefühl der Länder untermauert wird. 
Das Solidarprinzip sichert die finanzielle Leistungsfähigkeit der Länder unabhängig von ihrer Einnahmesituation und fördert das Gemeinschaftsgefühl unter den Ländern. Gerade dieses Gemeinschaftsgefühl, so Befürworter des Länderfinanzausgleichs, werde dadurch gefördert, dass Länder explizit von ihren Einnahmen an andere Länder abgeben. Die wiederholten Klagen der Geberländer vor dem Bundesverfassungsgericht widerlegen jedoch diese Einschätzung. Solidarität muss nicht erst wehtun, um zu nützen. Sowohl die vertikale Solidarität zwischen den Ebenen als auch die horizontale zwischen den Ländern wird von vornherein durch den großen Steuerverbund realisiert. Die Frage, wie die Einnahmen aus dem Steuerverbund verteilt werden, sollte dann nach pragmatischen Kriterien entschieden werden. Im aktuellen System wird zunächst sorgfältig auseinandersortiert, was jede Gebietskörperschaft an „eigenen“ Einnahmen erwirtschaftet hat. Dann wird auf Basis einer bestimmten Operationalisierung von „Finanzkraft“ (die auch anders ausfallen könnte, man denke nur an die heiße Debatte um den Anteil der Gemeindesteuern - 64 bis $100 \%$ - bei der Berechnung der Finanzkraft) in mehreren Schritten umverteilt, um diese Finanzkraft zwischen den Ländern weitgehend anzunähern. Grundsätzlich könnte ein solidarisches Verteilungssystem auch in einer umgekehrten Logik bestehen. Man könnte zunächst an alle Länder die Einnahmen so verteilen, dass sie bedarfsadäquat ausgestattet sind, und ihnen im zweiten Schritt erlauben, sich durch die Generierung eigener Einnahmen voneinander zu differenzieren. Im Falle sehr großer regionaler Disparitäten in den Ausgabelasten (insbesondere im Vollzug der Sozialgesetze) gibt es durchaus gute Argumente, diese Kosten zu vertikalisieren und dem Bund dafür einen größeren Anteil aus dem Steuerverbund zuzugestehen. Man würde sich dann die Mühe sparen, die Verteilung dieser Lasten in der Fläche abzuschätzen und durch Bedarfsindikatoren in der horizontalen Steuerverteilung abzubilden. Diese Frage soll hier nicht letztlich entschieden werden. Festzuhalten ist, dass Solidarität nicht nur durch Umverteilung, sondern auch durch Verteilung realisiert werden kann, und dass solidarische Verteilung nicht im Widerspruch zur Autonomie der Länder steht, also nicht zwangsläufig mit einer Vertikalisierung finanzieller Zahlungen einhergehen muss. 


\section{Die Definition des ,Eigenen“}

Die im bestehenden Finanzausgleichssystem gewählte Kombination von Autonomie- und Solidarprinzip äußert sich so, dass man sich zunächst bemüht, die Finanzkraft der Länder möglichst exakt zu ermitteln (Autonomie), um sie in weiteren Schritten weitgehend anzugleichen (Solidarität). Das Bemühen um exakte Ermittlung der Finanzkraft eines Landes folgt aus dem Grundsatz des örtlichen Aufkommens nach Art. 107 Abs. 1 GG. Er wurde vom Bundesverfassungsgericht so interpretiert, dass das Steueraufkommen möglichst nach ,wirklicher“ Steuerkraft verteilt werden soll, dass also „die Steuerleistung der Wirtschaft und der Bürger der des einzelnen Landes entspricht“" (BVerfGE 72, 330: C II 2 a). Es spricht vieles normativ dafür, den „wahren“ Ort des Entstehens der Wertschöpfung zu finden und den Mehrwert jenen Gebietskörperschaften zukommen zu lassen, die die Rahmenbedingungen für die Entstehung der Wertschöpfung bereitstellen. ${ }^{3}$ Denn offensichtlich gibt es Orte und Regionen, in denen mehr produziert wird als in anderen und die Arbeitskräfte und Investitionen anziehen. Und natürlich möchten diese Orte auch von ihrem Erfolg profitieren. Ähnlich wurde im vorangehenden Abschnitt zugunsten einer klaren Autonomie der Länder in vertikaler und in horizontaler Dimension argumentiert.

Sichtbarster Ausdruck des Bemühens um die zielgenaue Zuteilung des Steueraufkommens nach ,wirklicher" Steuerkraft ist die Steuerzerlegung der Einkommen- und Körperschaft- und auch der Zinsabschlagsteuer, die aber im Aufkommen weit weniger gewichtig ist (nach Art. 107 Abs. 2 GG sowie dem Zerlegungsgesetz - ZerlG vom 06.08.1998, BGB1. I S. 1998). Ziel der Steuerzerlegung ist es, Verzerrungen bei der Verteilung der Einkommen- und der Körperschaftsteuer zu korrigieren, die durch „,vielfältige Verflechtungen und Konzentrationsprozesse in der arbeitsteiligen Wirtschaft, namentlich durch das Auseinanderfallen von Unternehmenssitz und Betriebsstätten sowie durch die Einrichtung zentraler Lohnbüros" (BVerfGE 72, 330: C II 2 a) zustande kommen. Dementsprechend wird die Einkommensteuer (ESt) nach dem Wohnortprinzip, die Körperschaftsteuer nach dem Betriebsstättenprinzip zerlegt. Pendlerbewegungen, die

3 Abgesehen davon lässt sich das Prinzip des örtlichen Aufkommens aus dem demokratietheoretischen Grundsatz der institutionellen Kongruenz ableiten (vgl. Heinemann 2013, S. 40). 
insbesondere aber nicht nur die Stadtstaaten betreffen, sind hier eigentlich noch gar nicht mitgedacht. Allein die Zerlegung der Lohn- und Einkommensteuer führte $2010 \mathrm{zu}$ einer Bewegung von rund $10 \mathrm{Mrd}$. Euro zwischen den Ländern (vgl. Heinemann 2013, S. 41).

Die Steuerzerlegung illustriert aber auch sehr gut einen gewichtigen Einwand gegen die Idee der „wirklichen“ Steuerkraft: Durch pragmatische Entscheidungen von Bürgern oder Wirtschaftsunternehmen (nämlich aufs Land zu ziehen und in der Stadt zu arbeiten - im Falle von Bürgern - oder Lohnabrechnungsstellen oder Geschäftssitze zu zentralisieren - im Falle von Unternehmen) werden rein rechnerisch Steuereinnahmen für bestimmte Länder generiert, die offensichtlich nicht mit der realen Wertschöpfung vor Ort übereinstimmen. Diese Verzerrung soll dann korrigiert werden. Hierfür sieht das Zerlegungsgesetz vor, dass Lohn- und Einkommensteuer zu $100 \%$ dem Wohnsitzland des Steuerpflichtigen zu Gute kommen, die Körperschaftsteuer wird nach den an den einzelnen Betriebsstätten gezahlten Arbeitslöhnen zerlegt ( $\$ 2$ ZerlG mit Verweis auf $\S \S 28$ bis 31 sowie $\S 33 \mathrm{GewStG}$ ). Wie das Bundesverfassungsgericht ausdrücklich feststellt, wäre auch eine andere Zerlegungsregel verfassungskonform. Diese Beobachtung gilt generell für die Steuergesetze. Je nachdem, wie das Gesetz ausgestaltet ist, hat es gravierende Auswirkungen auf die Steuereinnahmen eines Landes. Die Steuereinnahmen, die von den Ländern als das „Eigene“, als Ausdruck ihrer politischen und wirtschaftlichen Leistungsfähigkeit und somit auch als ihnen moralisch zustehend angesehen werden, sind also massiv davon abhängig, wie die Gesetze den einzelnen Gebietskörperschaften Steuern oder Steueranteile zuerkennen. „Die Finanzminister der finanzstarken Länder in Deutschland meinen, wenn sie von ihren „eigenen“ Steuereinnahmen sprechen, das Steueraufkommen, das ihrem Haushalt gemäß den Regeln bezüglich der Feststellung des örtlichen Aufkommens und der Zerlegung im Bereich der Einkommensteuer-Teilsteuern zugerechnet wird. Ihnen ist wenig bewusst, dass diese Regeln ausgehandelten Konventionen entsprechen, das ihnen zugeordnete Steueraufkommen von der Art der ihnen zugeteilten Steuern und im Übrigen von der Steuerpolitik des Bundes determiniert ist" (Färber 2005, S. 150).

Wie kann aber ein Verteilungsergebnis, das sich als Artefakt ergibt, einen normativen Verfügungsanspruch begründen? Denn genau dies ist der psychologische Effekt: Die Fiktion, möglichst genau das „wirkliche“ Steueraufkommen zu ermitteln, suggeriert, dass dieses wirkliche Steueraufkommen diejenigen Finanzmittel seien, die einem Land eigentlich zu- 
stehen. Erhält es weniger, hat es von seinem „Eigenen“ etwas abgegeben. Die Zahlungen im Länderfinanzausgleich, aber auch die Differenz zwischen den einem Land tatsächlich zugewiesenen Umsatzsteueranteilen (die sich ggf. aus den Ergänzungsanteilen und der so genannten Restverteilung zusammensetzen) und dem Anteil, den es erhalten würde, wenn die Umsatzsteuer (USt) rein nach Einwohnern verteilt würde, werden sogar vom BMF in den jährlichen Endabrechnungen des Länderfinanzausgleichs ausgewiesen. Tatsächlich sind diese Differenzen aber irrelevant, da sie nur gegen eine fiktive Vergleichsgröße gerechnet werden, die normativ nicht besser begründet ist als die Summe, die sie tatsächlich erhalten. Psychologisch jedoch wird hierdurch großer Unmut erzeugt.

Weitere Argumente sprechen gegen einen normativen Verfügungsanspruch über das „eigene“ Steueraufkommen. Die bereits mehrfach erwähnten strategischen oder pragmatischen Unternehmensentscheidungen sowie die starke wirtschaftliche und finanzielle Verflechtung zwischen Gebietskörperschaften oder zwischen Wirtschaftsunternehmen setzen der Suche nach einer klaren örtlichen Radizierung der Wertschöpfung enge Grenzen. Auch die hohe Pfad- und Konjunkturabhängigkeit lässt den $\mathrm{Zu}$ sammenhang zwischen wirtschaftlicher Fähigkeit oder Kapazität und wirtschaftlichem Ertrag als wesentlich mittelbarer erscheinen als gemeinhin angenommen wird. Dasselbe gilt für Wirtschaftsunternehmen, die unterschiedlich erfolgreich am Markt agieren und dementsprechend unterschiedliche Gewinne erwirtschaften. Wenn diese erfolgreichen Unternehmer den Erfolg allein sich selbst zuschreiben, unterliegen sie demselben Irrtum wie Bayern, das von sich behauptet: „Die Landschaft ist ein Geschenk Gottes, alles andere ist harte Arbeit. "“4 So wie der erfolgreiche Unternehmer von der vom Staat bezahlten Arbeit der Kindergärtnerinnen und Grundschullehrerinnen profitiert, die die Kinder seiner Arbeitnehmer unterrichten und die er nicht selbst bezahlt, von den Straßen und Internetverbindungen, der guten Ärzteversorgung usw., so profitiert Bayern von den Universitäten, die für teures Geld bundesweit die Absolventen ausbilden, die dann nach Bayern kommen, um dort ihr Geld zu verdienen. Und es profitiert davon, dass in der ganzen Bundesrepublik BMWs gekauft werden und Weißbier getrunken wird. Der wirtschaftliche Erfolg eines Unternehmens oder eines Landes hängt sehr weitgehend von Rahmenbedingun-

4 So ein Wahlslogan der CSU und Lieblingsspruch von Peter Ramsauer. 
gen ab, auf die das Unternehmen oder das Land selbst wenig Einfluss haben. Insofern muss auch der Ertrag ihres Erfolges nicht notwendig ihnen selbst komplett zufließen, nur weil er bei ihnen erwirtschaftet wurde.

Zusammenfassend lässt sich also festhalten, dass das Bemühen, das „Eigene“ an Steuereinnahmen eines Landes möglichst passgenau festzustellen und zuzuweisen, nicht nur einen großen administrativen Aufwand mit sich bringt und auf gesetzlichen Regelungen beruht, die verfassungskonform auch ganz anders getroffen werden können. Darüber hinaus verkennt ein solches Zuteilungsverfahren die reale Interdependenz wirtschaftlicher Leistung und schafft psychologische Erwartungshaltungen und normative Anspruchsrechte, die bestenfalls mittelbar an eine zurechenbare Eigenleistung gekoppelt sind. In vielerlei Hinsicht wäre es daher empfehlenswert, die Bedeutung des „örtlichen Aufkommens“ von Steuern für die Steuereinnahmen eines Landes in den föderalen Finanzbeziehungen deutlich zu reduzieren.

\section{Finanzkraft oder strukturelle Bedarfe - das Problem regionaler Disparität}

Die Zuteilung von Steuermitteln nach Finanzkraft hat den Vorteil, dass hiermit ein scheinbar einfaches und objektives Kriterium gefunden ist, das darüber hinaus eine klare Koppelung an die Fähigkeit der Länder zur Erfüllung ihrer Aufgaben erlaubt. Die geringere Strategieanfälligkeit und relativ einfache Operationalisierung des Kriteriums gegenüber Bedarfsindikatoren führten unter anderem dazu, dass in der Schweiz in der großen föderalen Finanzreform von 2004 von einem stark bedarfsorientierten Verteilungssystem zu einem wesentlich finanzkraftorientierten Modus gewechselt wurde (Wettstein 2002). In den kommunalen Finanzausgleichssystemen der deutschen Länder hingegen findet sich eine Vielzahl von Bedarfsindikatoren, so dass wir hier in der Regel mit Mischsystemen zu tun haben. Auch der bundesdeutsche Finanzausgleich ist ja eigentlich ein Mischsystem, das zwar prinzipiell der Logik des Finanzkraftausgleichs folgt, aber mit einer Reihe von Bedarfselementen durchsetzt ist. Unter Umständen könnte es aber sogar besser sein, ganz auf einen Finanzkraftausgleich zu verzichten und sich bei der horizontalen Steuerverteilung ausschließlich an Bedarfen zu orientieren. Denn zwei gewichtige Argumente sprechen gegen einen Finanzkraftausgleich: 
Erstens ist das Kriterium gar nicht so unproblematisch und strategieresistent, wie es auf den ersten Blick erscheint. Die Diskussion im vorgängigen Abschnitt um die Zuordnung und Zerlegung der „eigenen“ Einnahmen, auf denen eine Berechnung der Finanzkraft notwendig basiert, zeigt, dass bereits die Berechnungsgrundlage der Finanzkraft problematisch ist. Aber auch die Operationalisierung ist nicht selbstevident. Das FAG in der geltenden Fassung sieht bereits zwei Formen der Berechnung der Finanzkraft vor. Zur Ermittlung der Ergänzungsanteile an der USt nach § 2 Abs. 1 FAG wird eine einfachere Berechnungsformel verwendet als zur Ermittlung der Ausgleichspflichtigkeit oder Ausgleichsberechtigung nach $\S \S 5-8$ FAG. Und die andauernde Diskussion darüber, zu welchem Prozentsatz die Finanzkraft der Gemeinden in die Finanzkraftmesszahl einzurechnen ist ( $88 \mathrm{Abs} .3 \mathrm{FAG}$ ), zeigt die hohe Strategieanfälligkeit der gewählten Operationalisierung.

Der zweite Einwand richtet sich gegen die implizite Folgerung, dass die Angleichung der Finanzkraft der angemessene Weg sei, die Länder auch tatsächlich in die Lage zu versetzen, ihre Aufgaben angemessen finanziell erfüllen zu können. Diese Folgerung trifft aber nur zu, wenn tatsächlich die Erfüllung der gleichen Aufgaben für alle Einwohner und in allen Landesteilen in etwa gleiche Kosten verursacht. Dass dies nicht so ist, ist weithin anerkannt. Im FAG selbst wird dem durch die unterschiedliche Einwohnergewichtung bei der Berechnung der Ausgleichsmesszahl ( 9 FAG) Rechnung getragen. Sonderlasten der Stadtstaaten, besonders hohe Bevölkerungsdichte sowie die Dünnbesiedelung der meisten ostdeutschen Länder werden durch diese Gewichtungsfaktoren abgebildet. Die unterschiedliche Einwohnergewichtung wurde in Verfassungsgerichtsentscheiden (BVerfGE 72, 330) sowie durch ein Gutachten des Bundesamtes für Bauwesen und Raumordnung wiederholt bestätigt (Eltges et al. 2001). Allerdings ist nicht gegen das Instrument der Einwohnerwertung an sich, sondern gegen die gewählte Umsetzung kritisch anzumerken, dass die Gewichtungsfaktoren sehr willkürlich gewählt sind und eine plausible Quantifizierung des Zusammenhangs zwischen struktureller Mehrbelastung und erhöhten Kosten vermissen lassen.

Neben akzeptierten Mehrbedarfen aufgrund unterschiedlicher Bevölkerungsdichte sowie einem Konglomerat von Faktoren, die zu einer spezifischen Belastung der Stadtstaaten führen, wurden in den vergangenen Jahren eine Reihe von Berechnungen angestellt, die verdeutlichen, dass insbesondere die Folgekosten der reformierten Sozialgesetzgebung für Länder und Kommunen sich regional sehr stark unterschiedlich verteilen 
(Eltges et al. 2001; Eck et al. 2014; Heinemann 2013; Knöchel et al. 2014). In Regionen mit einer erhöhten Dichte an sozialen Problemlagen fehlen dem Land oder der Kommune nicht nur Einnahmen aufgrund von Arbeitslosigkeit, sondern sie müssen außerdem erhöhte Ausgaben im Bereich der Sozialleistungen aufbringen.

Ein dritter Faktor, der noch nicht so gut mit Zahlenmaterial belegt ist, aber ein starker Kandidat für strukturelle Mehrbedarfe ist, ist der demographische Wandel (Seitz 2008). Gerade die dünn besiedelten Länder im Osten der Republik (vor allem Mecklenburg-Vorpommern und SachsenAnhalt) sind in besonders hohem Maß von Bevölkerungsrückgang, Abwanderung und Überalterung betroffen. Die überdurchschnittlich hohen Pro-Kopf-Ausgaben für Infrastruktur und Grundversorgung überlagern sich dort mit Rückbaukosten und teilweise gravierenden sozialen Problemlagen. Mindestens diese drei Faktoren - Dicht- bzw. Dünnbesiedelung, soziale Problemlagen und demographischer Wandel - begründen, dass die gleichen Leistungen pro Einwohner je nach Region ein Land unterschiedlich teuer kommen. Es ist daher sinnvoll, zwischen einem „Grundpreis“ pro Einwohner - gewissermaßen für ein standardisiertes Basispaket staatlicher Leistungen gemessen an Durchschnittswerten - und einem „erhöhten Preis" pro Einwohner zu unterscheiden, der in Relation zum Vorhandensein bestimmter Indikatoren für strukturelle Bedarfe auf den Grundpreis aufgeschlagen werden muss (vgl. Behnke 2012, S. 33ff.).

\section{Der Reformvorschlag}

Aus den bisherigen Ausführungen leitet sich der folgende Reformvorschlag ab:

- Das duale Steuersystem bleibt bestehen, in dem kleinere Steuern den Gebietskörperschaften direkt zugewiesen werden und im Land verbleiben, die aufkommensstarken Steuern in den Steuerverbund eingehen.

- An der vertikalen Steuerverteilung muss sich ebenfalls im Prinzip nichts ändern, es sei denn, bestimmte Ausgaben (bspw. Kosten der Unterkunft, Hilfen zur Erziehung u. a.) würden in Zukunft direkt vom Bund übernommen. Dann müsste ggf. die vertikale Steuerverteilung zugunsten des Bundes angepasst werden. Geeigneter Schalthebel für flexible Anpassung bleibt weiterhin die USt. 
- $\mathrm{Ab}$ der horizontalen Steuerverteilung ändert sich die Logik jedoch grundlegend. Der Länderanteil an den Verbundsteuern wird komplett nach Bedarfen auf die Länder verteilt. Hierbei ist eine Verteilungsformel zu finden, die den Anteil von Grundpreis pro Einwohner und strukturellen Sonderbedarfe angemessen quantifiziert und somit eine aufgabenadäquate Finanzausstattung der Länder unabhängig von ,eigenen" Einnahmen oder einer Berechnung ihrer Finanzkraft sichert. Die Datengrundlage, Standardisierung und Gewichtung der Indikatoren muss im Einzelnen durch Simulationen spezifiziert werden, wobei über die normativen Prämissen hierfür im politischen Diskurs Konsens erzielt werden muss. ${ }^{5}$

- Nachdem zuerst die Länder durch die bedarfsorientierte Verteilung der Gemeinschaftssteuern im Sinne des Solidarprinzips alle gleichermaßen in die Lage versetzt wurden, ihre Aufgaben mit der notwendigen finanziellen Ausstattung zu bestreiten, trägt man nachgeordnet nun dem Autonomieprinzip dadurch Rechnung, dass die Länder neue Steuererhebungskompetenzen zuerkannt bekommen, die es ihnen erlauben, auf länderspezifische Bedarfe einzugehen und sich von den anderen Ländern zu differenzieren. Diese Steuern verbleiben komplett im Land, in dem sie erhoben werden, und werden in keine Ausgleichsberechnung mit einbezogen.

- Weitere vertikale oder horizontale Ausgleichszahlungen sind in diesem System nicht mehr nötig.

$\mathrm{Zu}$ betonen ist, dass in diesem Reformvorschlag weiterhin beiden Komponenten, dem Autonomie- und dem Solidarprinzip, Rechnung getragen wird. Aber man würde die Verteilungslogik in der Reihenfolge vertauschen. Wird momentan viel Aufwand betrieben, um erst einmal nach dem Autonomieprinzip die eigenen Einnahmen der Länder zu bestimmen, daraus ihre Finanzkraft abzuleiten und diese dann in mehreren Schritten nach dem Solidarprinzip auszugleichen, würde nach dem Reformvorschlag zuerst den Ländern nach dem Solidarprinzip gewissermaßen ein „Grundeinkommen" zugewiesen, und davon unabhängig würden nach dem Autonomieprinzip bestimmte Steuern im Land erhoben, die vollständig im Land verbleiben. Die Vorteile eines solchen Systems bestehen darin, dass

5 Vgl. meinen Vorschlag zur Berechnung von Bedarfsindikatoren in Behnke (2012, S. $52 \mathrm{ff}$.). 
- die Einkommen-, Körperschaft- und Abgeltungsteuer nicht mehr horizontal zerlegt werden müssen;

- die USt nicht mehr in zwei Schritten zugeteilt wird, da nicht mehr zwischen Ergänzungsanteilen und Restverteilung nach Einwohnern unterschieden wird (vgl. zu dieser Kritik auch Wieland 2012, S. 42);

- die Finanzkraft der Länder nicht mehr berechnet werden muss, womit bspw. auch der Streit über das Ausmaß der Anrechnung der Kommunalsteuern sowie über den Prozentsatz der Einwohnerwertung wegfiele;

- keine weiteren horizontalen oder vertikalen Umverteilungsschritte mehr vorgenommen werden müssen, die ggf. die Solidarität zwischen den Ländern untergraben, da der Eindruck erweckt wird, Einnahmen, auf die man einen moralischen Anspruch habe, müssten abgegeben werden;

- Einnahmen, die klar nach dem Autonomieprinzip den Ländern zugeordnet werden, auch zu $100 \%$ im Land verbleiben.

Allerdings umreißt dieser Reformvorschlag bislang nur eine recht allgemeine Architektur eines Verteilungssystems. Viele Systemkomponenten müssen im Detail noch spezifiziert werden. Insbesondere drei Arten von Entscheidungen müssen getroffen werden, um das System zu optimieren. Im Folgenden werde ich diese drei Entscheidungen noch kurz diskutieren.

1. Entscheidung: Welche Steuern werden welcher Ebene zugeteilt, welche im Verbund erhoben?

Bei dieser Entscheidung muss im Prinzip keine vom Status quo abweichende Regel getroffen werden. Es ist jedoch angeraten, über die Sinnhaftigkeit der Zuerkennung bestimmter Steuern zu bestimmten Ebenen noch einmal nachzudenken. Die Überlegungen, aufkommensstarke Steuern im Verbund zu erheben, um eine große Verteilungsmasse zu schaffen, und diejenigen Steuern lokal zuzuordnen, die regionale Wirtschafts- und Finanzkraft abbilden, werden durch Argumente der Steuerinzidenz unterstützt. Auch nach dem Prinzip der Steuerinzidenz ist es naheliegend, aufkommensstarke Steuern im Verbund zu erheben und Steuern, die näherungsweise dem Äquivalenzprinzip genügen, auf niedrigeren Ebenen anzusiedeln (Färber 2005). Grundsätzlich könnten diese Überlegungen auch zu einer anderen Verteilung der Steuererhebungskompetenzen führen, als 
sie bislang besteht. Dies mag insbesondere für die Kommunen zutreffen, für die die Praktikabilität der Gewerbesteuer ja schon lange diskutiert wird.

2. Entscheidung: Verteilungsformel - Verhältnis von Grundpreis und erhöhten Preisen

In Abschnitt 4 wurden bereits Argumente dafür geliefert, dass insbesondere drei Faktoren strukturelle Mehrkosten pro Einwohner für die Länder bedingen können: die über- oder unterdurchschnittliche Bevölkerungsdichte, eine erhöhte Dichte sozialer Problemlagen sowie die demographische Entwicklung in einzelnen Regionen, namentlich der Bevölkerungsrückgang. Für diese Faktoren habe ich bereits an anderer Stelle Bedarfsindikatoren vorgeschlagen (Behnke 2012, S. 52ff.), an denen ich festhalten würde. Prinzipiell sollte also die Zuteilung der Länderanteile an den Gemeinschaftssteuern einer Verteilungsformel folgen, die die folgende Form aufweist:

$\operatorname{GemSt}(\mathrm{X})=\mathrm{a} * \mathrm{EZ}+\mathrm{b} * \mathrm{BD}+\mathrm{c} * \mathrm{SI}+\mathrm{d} * \mathrm{DE}$, mit

$\operatorname{GemSt}(\mathrm{X})=$ Zuweisung aus dem Länderanteil an den Gemeinschaftsteuern an das Land X;

$\mathrm{EZ}=$ Einwohnerzahl des Landes;

$\mathrm{BD}=$ Indikator für die Bevölkerungsdichte;

SI = Sozialindikator, also ein oder mehrere Indikatoren für soziale Problemlagen;

$\mathrm{DE}=$ Indikator für demographische Entwicklung;

a, b, c, d = Gewichtungsfaktoren für die einzelnen Indikatoren.

Für die Einwohnerzahl (EZ) kann man das bisherige Verfahren des Bundesfinanzministeriums der Finanzen (Stichtagswerte basierend auf den Daten des Statistischen Bundesamtes) fortführen.

Die Bevölkerungsdichte (BD) sollte im Sinne des erweiterten Brecht'schen Gesetzes als U-Kurve modelliert werden, also als beidseitige Abweichung vom Durchschnitt, wobei empirisch zu überprüfen wäre, ob beide Seiten der Kurve gleich steil ansteigen sollten. ${ }^{6}$ Verbessert werden könnte dieser Indikator, wenn es gelänge, die Abweichungen von der durchschnittlichen

6 Vgl. die Ausführungen zur Brechtkurve im Gutachten des Bundesamtes für Bauwesen und Raumordnung (Eltges et al. 2001, S. 17 ff.) 
Bevölkerungsdichte nicht nur landesweit zu berechnen, sondern als Summe aus den Werten kleinerer Regionen, um zu vermeiden, dass sich innerhalb eines Landes regionale Problemlagen, die sich einerseits durch Ballungszentren und andererseits durch Dünnbesiedelung ergeben, ausmitteln. ${ }^{7}$ Man könnte die Bevölkerungsdichte direkt auf Gemeindeebene erheben und würde so einen sehr feingliedrigen Indikator erhalten. Alternativ wäre auch denkbar, Arbeitsmarktregionen als Basis zur Berechnung der Bevölkerungsdichte heranzuziehen. Hierfür stellt das Bundesamt für Bau-, Stadt- und Raumforschung (BBSR) regelmäßig Daten zur Verfügung. ${ }^{8}$

Ein guter Indikator für soziale Problemlagen ist nach wie vor die Arbeitslosigkeit (AL), die mit allen anderen Kennziffern für soziale Problemlagen hoch korreliert. Denkbar wäre aber auch hier die Kombination mit weiteren Indikatoren, bspw. der Anzahl der SGBII-Bedarfsgemeinschaften, wie im Reformvorschlag der LINKEN (Knöchel et al. 2014, S. 29) angeregt wird.

Für die demographische Entwicklung (DE) schließlich würde ich mich weiterhin am Indikator „Bevölkerungszunahme $(+)$ bzw. Bevölkerungsabnahme (-) je 10.000 Einwohner" orientieren, der im Regionalatlas des statistischen Bundesamtes ausgewiesen wird (vgl. Behnke 2012, S. 62).

Die Gewichtungskoeffizienten, mit denen die einzelnen Faktoren in die Verteilungsformel eingehen, können durch Simulationen gewonnen werden, die auf der Basis politischer Vorgaben Verteilungsgewichte optimieren.

Andere Bedarfe, die auf einer Ad hoc-Basis im bestehenden FAG berücksichtigt werden, sind die Kosten der politischen Führung und die nachholenden Investitionskosten in Folge der Wiedervereinigung in den ostdeutschen Ländern (beides wird in den Sonder-Bundesergänzungszuweisungen (SoBEZ) durch diskrete, jährlich degressiv ausgestaltete Zahlungen abgebildet). Für den Sonderbedarf in Folge der Kleinheit der politischen Einheit gibt es keine solide Berechnungsgrundlage, die Folgekosten der Wiedervereinigung insbesondere im Bereich der nachholenden In-

7 Dieses Argument wird bspw. häufig für Hessen vorgebracht, das im Großraum Frankfurt Ballungszentrumslasten zu tragen hat, aber im Norden des Landes sehr dünn besiedelte Regionen aufweist.

8 http://www.bbsr.bund.de/BBSR/DE/Raumbeobachtung/Raumabgrenzungen/AMR _2011/Arbeitsmarktregionen html, letzter Aufruf 2.6.2014. 
vestitionen und Infrastrukturausbauten dürften 2020 weitgehend ausgeglichen sein. Beide stellen keine strukturellen Bedarfe dar, die erhöhte Kosten pro Einwohner für die Bereitstellung staatlicher Leistungen generieren. Daher sollten diese Sonderbedarfe nicht weiterhin in einer bedarfsorientierten Steuerverteilung aufgenommen werden.

3. Entscheidung: Wo werden Fiskalkompetenzen der Länder ausgeweitet, um regionale Leistungsdifferenzierung zu erlauben?

Wäre es gelungen, durch eine Formel zur horizontalen Verteilung des gesamten Länderanteils an den Gemeinschaftsteuern, die Grund- und strukturelle Sonderbedarfe angemessen abbildet, alle Länder so hinreichend mit Finanzmitteln auszustatten, dass sie alle gleichmäßig in die Lage versetzt sind, ihre Aufgaben zu erfüllen, bliebe im nächsten Schritt die Möglichkeit, durch länderspezifische Steuerfindungs- oder Hebesatzrechte eine Differenzierung der Ländereinnahmen nach der Zahlungsfähigkeit der Bürger und Wirtschaftssubjekte vorzunehmen. Die Frage ist dann aber, welche Steuer sich hierfür eignen würde und wie die Steuerkompetenzen der Länder entsprechend ausgestaltet werden sollten. Eine länderspezifische Steuer, die zwischen den Ländern mit unterschiedlichen Steuersätzen ausgestaltet werden kann, muss einige Kriterien erfüllen.

- Sie müsste ein nicht zu mobiles Steuerobjekt besteuern, um hebesatzinduzierte Wanderungsbewegungen zu vermeiden.

- Sie müsste klar örtlich zurechenbar sein (Problem bei Unternehmenssteuern ist ja gerade die Möglichkeit mehrerer Betriebsstätten).

- Sie müsste einigermaßen ertragreich sein.

- Sie müsste im Sinne des Äquivalenzprinzips eine Kopplung von staatlicher Leistung und Steuerertrag herstellen.

- Es dürfte grundsätzlich nur einen Hebesatzkorridor geben, um von vornherein ein „Race to the Bottom“ und ein „Race to the Top“ zu verhindern.

Eine Möglichkeit, diese Prinzipien zu verwirklichen, wäre ein länderspezifischer Zuschlag auf die Einkommensteuer, wie sie bspw. auch in den aktuellen Reformvorschlägen für das britische Finanzzuweisungssystem diskutiert wird. Die Frage, wie mobil der Lohn- oder Einkommensteuerzah- 
lende zwischen Bundesländern wandern würde, um ggf. der Steuerlast auszuweichen, ist empirisch nicht geklärt. Zweifelsfrei sind jedoch andere Faktoren als der Steuersatz (die Verfügbarkeit eines Arbeitsplatzes, familiäre Bindungen, Bindungen an ein bestehendes soziales Umfeld) relevanter für die Wahl des Arbeitsortes als der Steuersatz. Insofern könnte man annehmen, dass Bedingung 1 näherungsweise erfüllt ist. Ein Problem ist Bedingung 2, denn mit einem Länderzuschlag auf die Einkommensteuer würde man sich wieder die Zerlegungsproblematik einhandeln. Eine mögliche Lösung bestünde darin, dass der Zuschlag jeweils zur Hälfte dem Wohnsitz- und dem Arbeitsplatzland zu Gute kommt. Bedingung 3 ist erfüllt. Bedingung 4 ist näherungsweise erfüllt, da das Land mit seinen Leistungen für Einwohner und Wirtschaftsunternehmen die Rahmenbedingungen zur Verfügung stellt, um Einkommen zu erwirtschaften. Und Bedingung 5 könnte man dadurch erfüllen, dass man bspw. den bundesweiten Einkommensteuertarif um 3 Prozentpunkte absenkt, so dass der Eingangssteuersatz dann entsprechend aktuell bei $12 \%$ anstatt bei $15 \%$, der Spitzensteuersatz bei $39 \%$ anstatt bei $42 \%$ läge (respektive bei $42 \%$ anstatt $45 \%$ ab 250.000 Euro). Nun erhielten die Länder das Recht, einen $\mathrm{Zu}$ schlag auf die ESt zu erheben, der zwischen 0 und 5 Prozentpunkte liegen dürfte. Somit wäre die Gefahr eines Dumpingwettbewerbs durch die Vorgabe eines Korridors begrenzt, aber die Länder hätten einen Entscheidungsspielraum bezüglich der Höhe der zusätzlichen Steuereinnahmen und könnten mit diesem Geld politische Schwerpunkte setzen. Gemäß dem für Punkt 4 vorgeschlagenen Zerlegungsprinzip könnte man so vorgehen, dass bei Berufspendlern das Wohnsitzland die Hälfte seines geltenden Aufschlags erheben darf und das Arbeitsplatzland ebenfalls. Pendelt bspw. ein Arbeitnehmer von seinem Wohnort in einem Bundesland, wo der länderspezifische Aufschlag auf die ESt 4 Prozentpunkte beträgt, zu seinem Arbeitsplatz in ein Land, wo der länderspezifische Aufschlag auf die ESt 2 Prozentpunkte beträgt, dann müsste er im Wohnortland einen Aufschlag von 2 Prozentpunkten entrichten und im Arbeitsplatzland einen Aufschlag von 1 Prozentpunkt. Das wäre immer noch rechnerisch und administrativ einigermaßen einfach umzusetzen.

Eleganter wäre tatsächlich eine Steuer, die keine Zerlegungsproblematik aufweisen würde, wie bspw. die Grundsteuer, die man auf diese Weise auch wieder in ihrer Ertragsstärke den europäischen Nachbarländern entsprechend aufwerten könnte. Andererseits sprechen ansonsten viele Argumente für einen Zuschlag auf die Einkommensteuer. Die Höhe des Ab- 
schlags auf den bundesweiten Tarif sowie der Umfang des Korridors müssten wieder im Rahmen von Simulationen geschätzt werden.

\section{Folgewirkungen der hier vorgeschlagenen Reformen}

Der hier vorgelegte Reformvorschlag würde in seiner Umsetzung die bestehende Architektur der föderalen Finanzbeziehungen sehr weitgehend verändern. Es gibt grundsätzlich sehr gute Gründe, bei komplexen Systemen Reformen tendenziell eher inkrementalistisch vorzunehmen, um großen negativen nicht-intendierten Folgewirken vorzubeugen (Hayek 2003 [1967]). Andererseits kann gerade das System der föderalen Finanzbeziehungen nur durch eine sehr weitgehende Reform auf eine andere Logik umgestellt werden. Ich bin überzeugt, dass der hier präsentierte Vorschlag sehr viel Rationalität in die Steuerverteilung bringen könnte und auch die jahrzehntelangen Streitigkeiten weitgehend ausräumen könnte. Denn die hier vorgeschlagenen Bedarfsindikatoren sind relativ wenig strategieanfällig und in der Debatte weitgehend unstrittig. Die wiederholt vorgebrachten Streitpunkte fokussieren eher auf die Berechnung der Finanzkraft (Einwohnerwertung, Gemeindeanteil) und auf die umverteilenden Eingriffe in den (zumindest psychologisch so wahrgenommenen) eigenen Steuerbestand der Geberländer durch die Ergänzungsanteile der USt und den horizontalen Finanzausgleich. Der Sorge derjenigen Länder, die eine stark unterdurchschnittliche Finanzkraft aufweisen, wird durch die Verteilung der Steueranteile nach Einwohnern und Sonderbedarfen Rechnung getragen. Und die Sicherung eigener Einnahmen auf Basis einer erweiterten Fiskalautonomie der Länder (z. B. durch einen Aufschlag auf die Einkommensteuer) trägt dem Autonomiebedürfnis der Länder Rechnung.

Der Einwand vieler Gegner großer Reformen, dass diese ja eine Änderung des Grundgesetzes nötig machen würden, ist richtig, aber nicht gewichtig. Die Finanzverfassung ist ohnehin einer der am häufigsten geänderten Teile des Grundgesetzes, was nicht zuletzt daran liegt, dass hier eine Fülle von Detailregelungen ihren Weg in die Verfassung gefunden haben, die sinnvollerweise besser durch einfachgesetzliche Regelungen festgelegt und geändert würden. Konkret müsste Art. 107 weitgehend geändert oder gestrichen werden. Art. 107 Abs. 1 S. 1-3 bestimmen das Prinzip des örtlichen Aufkommens und der Zerlegung. S. 4 regelt die aktuelle USt-Verteilung. Art. 107 Abs. 2 verlangt einen horizontalen Finanzausgleich unter Bezug auf Finanzkraft der Länder und erlaubt Ergänzungszu- 
weisungen des Bundes. Im Sinne einer Rückgewinnung an Rationalität wäre dann auch angeraten, Art. 106 zu überarbeiten. Art. 106 Abs. 3 S. 5 und entsprechend Art. 106 Abs. 4, wo auf Abs. 3 Satz 5 Bezug genommen wird (Berücksichtigung von Steuermindereinnahmen bei der vertikalen Steuerverteilung), sowie Art. 106 Abs. 5a, Art. 106a und Art. 106b enthalten durchweg Sonderregelungen, die eigentlich im GG nichts zu suchen haben. Sofern sie fortbestehen, sollten sie im FAG direkt und nicht im GG geregelt werden.

Eine Neujustierung der Gesamtarchitektur der föderalen Finanzbeziehungen kann langfristig nur dann tragfähig sein, wenn erstens parallel dazu eine Lösung für die Altschuldenproblematik gefunden wird. Gewissermaßen stellen auch Altschulden strukturelle Sonderbedarfe dar, die historisch ererbt sind und sich dem unmittelbaren politischen Einfluss der Regierung des Tages entziehen. Diese Problematik aber im Rahmen des Finanzausgleichssystems zu lösen, würde dieses im Volumen schlicht überfordern. Mitdenken muss man zweitens die finanziellen Verflechtungen im Sozial- und Bildungsbereich. Wie die Diskussionen und fortschreitende finanzielle Inpflichtnahme des Bundes über Geldleistungsgesetze, etwa bei den Kosten der Unterkunft, der Grundsicherung im Alter oder der Wiedereingliederungshilfe zeigen, fallen hier Kosten an, die nur solidarisch zwischen den Ebenen bewältigt werden können. Ob hierfür Art. 104a immer das geeignete Instrument ist, ist fraglich, da bei einer Beteiligung des Bundes an den Ausgaben, die $50 \%$ übersteigt, aus einem Mischfinanzierungstatbestand eine Bundesauftragsverwaltung wird (Art. 104a Abs. 3). Ähnliches gilt für Ausgaben im Bildungsbereich. Die Länder realisieren zunehmend, dass sie sich mit dem im Zuge der Föderalismuskommission I erkämpften Kooperationsverbot selbst ein Bein gestellt haben. Die verfassungsrechtlichen Klimmzüge zur Finanzierung des Kindertagesstättengesetzes illustrieren die Irrationalität und Praxisferne der geltenden Regelung. Ähnliches gilt für den Bereich der Hochschulbildung. Nicht nur der Neu- und Ausbau von Hochschulbauten, der als Gemeinschaftsaufgabe ebenfalls im Zuge der Föderalismuskommission I 2006 abgeschafft wurde (Art. 91b alte Fassung), sondern auch der laufende Betrieb sind Kosten, die vom Umfang und den externen Effekten her nach dem Subsidiaritätsprinzip eine finanzielle Einbeziehung des Bundes erfordern würden. Insofern müsste auch hier überlegt werden, wie man diese Problematik systematisch in die föderale Finanzverteilung mit einbezieht. Drittens ist die logische Folgerung aus der hier vorgeschlagenen fundamentalen Neujustierung der Komponenten Autonomie und Solidarität die 
Überlegung, ob die vertikale Verteilung der Gemeinschaftssteuern nicht auch auf der kommunalen Ebene noch einmal neu sortiert werden müsste. Schon lange ist die Gewerbesteuer als wichtigste autonome Einnahmequelle der Kommunen in der Kritik, und gerade die Finanzverteilungssysteme zwischen Land und Kommunen im Rahmen des kommunalen Finanzausgleichs stellen aufgrund des Kooperationsverbots aktuell eine relevante Hürde für die Kofinanzierung kommunaler Sozialleistungen durch den Bund dar. Auch dieser Aspekt muss bei einer Reform der föderalen Finanzbeziehungen sorgfältig mit bedacht werden. Andererseits wäre es eine Gelegenheit, auch dieses Dauerproblem, das sich einer Lösung über eine Vielzahl von Reformkommissionen hinweg hartnäckig widersetzt, einer größeren Rationalität zuzuführen.

Die hier präsentierten Überlegungen sind eine konsequente Fortentwicklung des ersten Reformvorschlags, den ich zwischen 2010 und 2012 erarbeitet habe. Es handelt sich hierbei noch nicht um ein im Detail ausgefeiltes Regelwerk, sondern um ein Konzept für eine neue Architektur. Die Details müssen im Zuge von Simulationsrechnungen präzisiert werden. Zugleich sehe ich den Vorschlag als eine Einladung zur Diskussion über die Prinzipien, die unserem Finanzausgleichssystem zugrunde liegen. Denn nach wie vor sehe ich das Verfallsdatum von 2019 als Chance, sich von einem weitgehend an Besitzstandswahrung orientierten Inkrementalismus zu lösen und im besten Sinne politisch darüber nachzudenken, auf welchen Ordnungsprinzipien das föderale Miteinander basieren sollte.

\section{Literatur}

Bakvis, Herman, Baier, Gerald und Brown, Douglas M., 2009, Contested Federalism. Certainty and Ambiguity in the Canadian Federation, Don Mills, Ontario: Oxford University Press.

Behnke, Nathalie, 2012, Fahrplan für eine grüne Reform der bundesdeutschen Finanzverfassung, Konstanz.

Eck, Alexander, Ragnitz, Joachim, Rösel, Felix, Steinbrecher, Johannes und Thater, Christian, 2014, Wer bestellt, bezahlt, Bonn: Friedrich-Ebert-Stiftung, Wiso direkt.

Eichel, Hans, Fink, Philipp und Tiemann, Heinrich, 2013, Vorschlag zur Neuordnung des Finanzausgleichs, Bonn: Friedrich-Ebert-Stiftung, Wiso direkt.

Eltges, Markus, Zarth, Michael, Jakubowski, Peter und Bergmann, Eckhard, 2001, Abstrakte Mehrbedarfe im Länderfinanzausgleich, Bonn: Bundesministerium des Innern, Bundesamt für Bauwesen und Raumordnung. 
Färber, Gisela, 2005, Die regionale Inzidenz des deutschen Steuersystems, in: Gisela Färber (Hrsg.), Das föderative System in Deutschland: Bestandsaufnahme, Reformbedarf und Handlungsempfehlungen aus raumwissenschaftlicher Sicht, 146-173, Hannover: Verlag der ARL.

Feld, Lars P., Kube, Hanno und Schnellenbach, Jan, 2013, Optionen für eine Reform des bundesdeutschen Finanzausgleichs.

http://www.eucken.de/fileadmin/bilder/Dokumente/Gutachten_Finanzausgleich.pdf: Gutachten im Auftrag der FDP-Landtagsfraktionen der Länder Baden-Württemberg, Bayern und Hessen.

Hayek, Friedrich August von, 2003 (1967), Die Ergebnisse menschlichen Handelns, aber nicht menschlichen Entwurfs, in: Friedrich August von Hayek (Hrsg.), Rechtsordnung und Handelnsordnung. Aufsätze zur Wirtschaftsökonomik, 178-198, Tübingen: Mohr (Siebeck).

Heinemann, André, 2013, Finanzströme in Deutschland - Ein Überblick zu den finanziellen Verflechtungen im Mehrebenensystem der Bundesrepublik Deutschland, Bremen: Gutachten im Auftrag der Landtags-Fraktionen von Bündnis 90/DIE GRÜNEN in den Ländern Berlin, Bremen, Hamburg, Mecklenburg-Vorpommern, Niedersachsen, Sachsen-Anhalt und Schleswig-Holstein.

Hesse, Konrad, 1962, Der unitarische Bundesstaat, Karlsruhe: C. F. Müller.

Knöchel, Swen, Restat, Jan, Schmidt, Manuela, Schuster, Andreas, Troost, Axel und Würzbach, Stefan, 2014, Länderfinanzausgleich LINKS gedacht: sozial und solidarisch - Ein Diskussionsangebot. http://dokumente.linksfraktion.de/inhalt/landerfifinanzausgleichlinks-gedacht-sozial-solidarisch-21-03-14.pdf:

Korioth, Stefan, 2009, Zur Neuordnung des Finanzausgleichs zwischen Bund und Ländern - Vertikalisierung und Bedarfstatbestände als mögliche Auswege aus der verfahrenen Verhandlungskonstellation?, in: Ralf Thomas Baus, Henrik Scheller und Rudolf Hrbek (Hrsg.), Der deutsche Föderalismus 2020, 195-203, BadenBaden: Nomos.

Lehmbruch, Gerhard, 2002, Der unitarische Bundesstaat in Deutschland. Pfadabhängigkeit und Wandel, in: Arthur Benz und Gerhard Lehmbruch (Hrsg.), Föderalismus. Analysen in entwicklungsgeschichtlicher und vergleichender Perspektive, 53110, PVS Sonderheft 32/2001: Opladen: Westdeutscher Verlag.

Seitz, Helmut, 2008, Die Demographieabhängigkeit der Ausgaben und Einnahmen der öffentlichen Haushalte. Eine empirische Analyse unter Berücksichtigung der föderalen Verflechtungen, Gütersloh: Bertelsmann-Stiftung.

Trench, Alan, 2013, Funding devo more, London: IPPR.

Wettstein, Gérard, 2002, Die Neugestaltung des Finanzausgleichs und der Aufgaben (NFA) - Erfolgsfaktoren und Hürden aus Sicht der Projektleitung, LeGes 13: 35-42.

Wieland, Joachim, 2012, Neuordnung der Finanzverfassung nach Auslaufen des Solidarpakts II und Wirksamwerden der Schuldenbremse, Gütersloh: Bertelsmann Stiftung. 